\title{
Impact on Power Consumption of Zigbee based Home Automation Network using Various Traffic
}

\author{
Gurjit Kaur $^{1}$, Kunal Malik ${ }^{2}$ and Kiran Ahuja ${ }^{3}$ \\ ${ }^{1}$ Student, ECE, DAVIET Jalandhar, Punjab, India \\ ${ }^{2}$ Student, ECE, HCTM Kaithal, Kurukshtera, Harayana. India \\ ${ }^{3}$ Assistant Professor, ECE, DAVIET Jalandhar, Punjab. India \\ Iguri_23s@yahoo.co.in, ${ }^{2}$ kunalmalik4@gmail.com,33askahuja2002@yahoo.co.in
}

\begin{abstract}
Wireless sensor networks are power constrained since nodes operate with limited battery energy. In order to maximize the lifetime of sensors networks, traffic should be sent via a route and different traffic applications that can be avoid nodes with low energy. In this paper, we analyze the performance of Zigbee home automation network under different traffic applications (such as constant bit rate (CBR), variable bit rate (VBR), and file transfer protocol $(F T P))$ by considering energy consumption parameter. The simulation results represent the impact on power consumption due to these different traffic applications in transmit mode, receive mode and idle mode. Energy consumed in transmits, receive and idle mode, for FTP application is low as compare to VBR and CBR. In terms of energy consumption FTP overall performs better than other traffic application since low power consumption corresponds to high efficiency.
\end{abstract}

Keywords: CBR, VBR, FTP, WPAN, MAC, PAN

\section{Introduction}

The emergence of wireless sensor networks (WSNs) enabled the development and subsequently the release of IEEE 802.15.4 [1] standard for low-rate wireless personal area networks (LRWPANs). Zigbee [2], which has MAC/PHY layers of IEEE 802.15.4, is a protocol for well-known sensor nodes, its most frequently cited characteristics are low power consumption, long battery life, low product cost, and open standards. Mesh networking of Zigbee also makes IEEE 802.15.4 attractive in home automation. In order to install this wireless network in home networks and meet a variety of the application requirements of the home automation, it should be able to guarantee time constrained behaviour and provide priority to nodes or packets. Satisfying these requirements is closely related to the contention for medium access between nodes, a beacon frame period, and sleep/wake duration in LRWPAN [3].

One of the goals of Zigbee is to control and monitor environment using aggressive low energy transmission techniques so as to allow continuous use without changing battery for six months to two years $[4,5]$.

This paper provides the performance analysis of different traffic application like CBR (Constant Bit Rate), VBR (Variable Bit Rate) and FTP (File Transfer Protocol) and their impact on power consumption on Zigbee network based home automation system in Qualnet using static IEEE802.15.4 star topology. The impact on power consumption by different 
traffic applications are measured in physical layer of Zigbee network based home automation scenario.

The organization of the paper is as follows: Section 2 represented related work to IEEE 802.15.4 in various simulation environments. The overview of Zigbee and IEEE 802.15.4 protocol is discussed briefly in Section 3. Simulation set up has been discussed in Section 4. Simulation results have been discussed in Section 5. Finally, we conclude our work in Section 6.

\section{Related Work}

According to our best knowledge, there exist only few articles that analyze mathematically or simulate the performance of IEEE 802.15.4. The performance of IEEE 802.15.4 in a star network with 100 nodes was analyzed in $[6,7]$. The paper contained a compact mathematical analysis of average power consumption and transmission failure rate. The analysis was complemented with real measurements of steady state powers and transient energy, and switch times from a standard compliant evaluation board. A special contribution was bit error rate measurements with two evaluation boards connected through a set of calibrated attenuators. The operational analysis considered the effect of path loss and packet size on energy consumption.

In [8], implemented the IEEE 802.15.4 standard on NS2 simulator and provided the comprehensive performance evaluation on 802.15.4. In [9] provided performance evaluations of IEEE 802.15.4 MAC in becon-enabled mode for a star topology. The performance evaluation study revealed some of the key throughput-energy-delay tradeoff inherent in IEEE 802.15.4 MAC.

The performance of IEEE 802.15.4 was analyzed for medical sensor body area networking in [10]. The analysis considered quite extensively a very low data rate star network with 10 body implanted sensors transmitting data 1 to 40 times per hour. The analysis focused on the effect of crystal tolerance, frame size, and the usage of IEEE 802.15.4 Guaranteed Time Slots (GTS) on a node lifetime. For analyzing the standard performance in WSN applications, further analysis with larger and more complex network topologies and other IEEE 802.15.4 MAC parameters would be required.

In [11] presented a novel mechanism intended to provide Quality of Service (QoS) for IEEE 802.15.4 based Wireless Body Sensor Networks (WBSN) used for pervasive healthcare applications. The mechanism was implemented and validated on the Aquis Grain WBSN platform.

The performance simulations of IEEE 802.15.4 in a star network were presented in [12]. The network consisted of 49 nodes configured to IEEE 802.15.4 beacon-enabled mode. The evaluation considered latency and energy with different amounts of background traffic. Also, the performance of IEEE 802.15.4 GTS and beacon tracking were simulated. Still, the applicability of the results for WSN applications was insufficient, since larger network sizes with cluster tree network topologies were required.

\section{Zigbee Wireless Network}

The 802.15.4 standard defines physical (PHY) and medium access control (MAC) layer protocols for supporting relatively simple sensor devices that consume minimal power and operate in an area of $10 \mathrm{~m}$ or less. The point of service (POS) may be extended beyond $10 \mathrm{~m}$ but this requires additional energy to operate. It also allows two types of topologies such as a simple one hop star or a self configuring peer-to-peer network to be established. In terms of wireless links, 802.15.4 operates in three license free industrial scientific medical (ISM) 
frequency bands, i.e., data rates of $250 \mathrm{kbps}$ in the $2.4 \mathrm{GHz}$ band, $40 \mathrm{kbps}$ in the $915 \mathrm{MHz}$ band, and $20 \mathrm{kbps}$ in the $868 \mathrm{MHz}$ band. The first band has 16 channels while the second has 10 .

The latter was allocated one channel. Though only one channel is used at a time, the additional channels allow the flexibility of switching to another in case the existing becomes not conducive [13].

There are two categories of devices in 802.15.4. One of them is called full-function device (FFD) while the other is reduced-function device (RFD). RFD is crude device supporting simple application such as a switch or sensor. It is usually controlled by FFD device. RFDs can be used to communicate among themselves and with FFDs. The former is desired in this paper because it can take on the role of a router that enables peer-to-peer communication. In terms of addressing, the protocol assumes the use of either 16bit short or extended 64-bit IEEE addresses. The latter is available in all devices by default and is commonly known as physical (MAC) address while the previous is allocated by the PAN coordinator. In the following section we shall describe briefly the IEEE 802.15.4 standard particularly the PHY and MAC layer [14].

\subsection{Physical Layer}

The PHY layer provides an interface between the MAC sub layer and the physical radio channel. It provides two services, accessed through two service access points (SAPs). These are the PHY data service and the PHY management service. The PHY protocol performs energy detection (ED) scan and clear channel assessment (CCA) on the channel to detect any ongoing activities and relay the results to the MAC layer. A channel is considered busy if the activity levels detected exceed certain threshold value. Another important assessment is link quality.

Upper layers protocols (MAC and network) depend on link information before deciding on using a particular channel because external interferences such as noise and electromagnetic signal could affect the network performance. If a particular channel is not feasible, there are 26 other channels available under 802.15.4 to be selected. As part of 802.15.4 effort in preserving energy, the radio transceiver can be turned off if inactive (not receiving or transmitting).

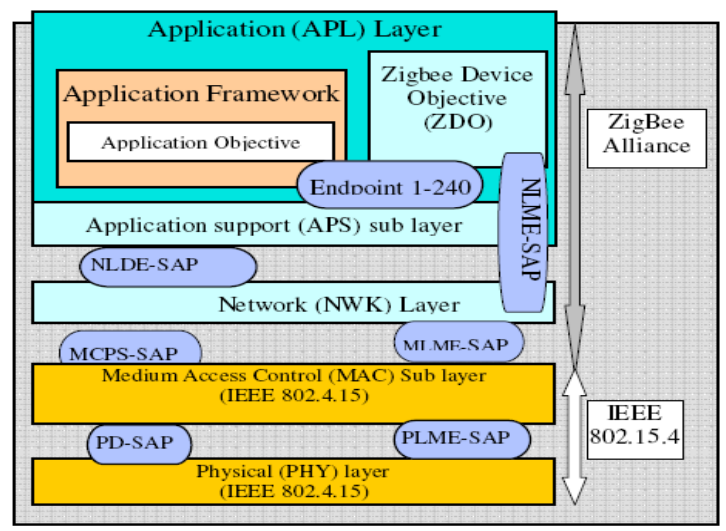

Figure 1. Zigbee Functional Layer Architecture and Protocol Stack [14]

\subsection{MAC Layer}


This layer provides an interface between upper layers and the PHY layer. It handles channel access, link management, frame validation, security, and nodes synchronization. In our approach, we adopt beaconless mode which implies un-slotted CSMA/CA mechanism. For this mode, the PAN coordinator is responsible of handling only device association/disassociation and (short) address allocation in case the 64-bit IEEE addressing is not used.

The CSMA/CA protocol is an important mechanism for channel access but does not include the RTS/CTS handshake, considering low data rate adopted in 802.15.4. This mechanism evaluates the channel and allows data packets to be transmitted if the condition is suitable (free of activities). Otherwise the algorithm shall back off for certain periods before assessing the channel again. Without the RTS/CTS handshake, it would appear to encourage packet collisions due to hidden nodes [15]. Nodes are considered hidden if they are out of signal range of each other. By using the above discussion we setup a system for home automation using 802.15.4 standard in the following section.

\section{Simulation Setup}

The main objective of this paper is to analyze the effect of different traffic applications like CBR, VBR and FTP on the performance of Zigbee network based home automation system using static IEEE 802.15.4 star topology. The simulations have been carried out using Qualnet version 5.0.

In the simulation model, a star topology with one PAN co-ordinator, one PDA (personal digital assistants), and 10 devices are uniformly deployed in an area of $1500 \mathrm{~m} \times 1500 \mathrm{~m}$. PAN is static mains powered device placed at the centre of the simulation area. Only the uplink traffic i.e., devices to PAN co-ordinator are considered in the simulations which suits WSN application like automation industry where a large number of devices communicates to a single sink server for data delivery and processing.

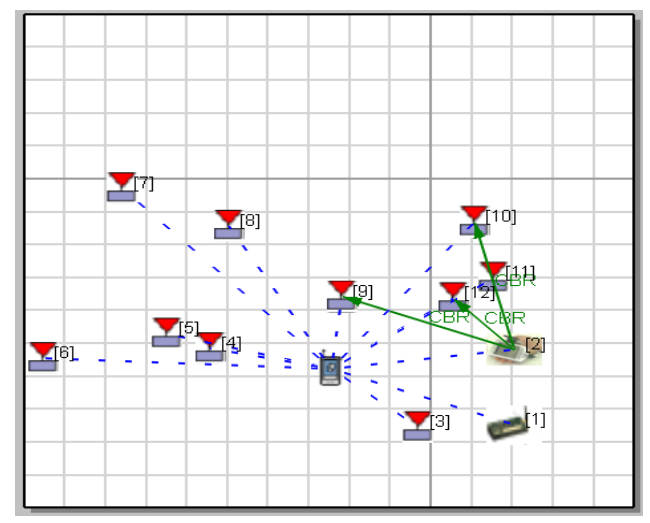

Figure 2. Simulation Setup of Zigbee Network based Home Automation System

The transmission range of devices is one hop away from PAN Coordinator in star topology. The fact that $\mathrm{BO}$ (Beacon order) $=\mathrm{SO}$ (super frame order) assures that no inactive part of the super frame is present [16]. A low value of this parameter implies a great probability of collisions of beacon frames as these would be transmitted very frequently by coordinators. On the contrary, a high value of the BO (beacon order) introduces a significant delay in the time required to perform the MAC association procedure since channel duration which is a part of association procedure is proportional to BO (beacon order). In our simulation model, function for acknowledging the receipt of packets is disabled. It is due to 
the fact that overhead mechanism is too expensive for low data rate WSN application for which 802.15.4 is designed.

Impact on energy consumption of Zigbee network based home automation system is done by applying different traffic types. Where, energy consumption is defined as amount of energy consumed by mobile devices during the periods of transmitting, receiving, idle and sleep. The unit of energy consumption used in the simulations is mJoule. The metric is measured as the percent of energy consumed by a node with respect to its initial energy. The initial energy and the final energy left in the node, at the end of the simulation run are measured. The percent energy consumed by a node is calculated as the energy consumed to the initial energy. Finally the percent energy consumed by all the nodes in a scenario is calculated as the average of their individual energy consumption of the nodes.

Energy Consumed $(\%)=\frac{\text { Initial Energy }- \text { Final Energy } \times 100}{\text { Initial Energy }}$

Sum of Percent Energy Consumed by All

Average Energy Consumed =

Number of Nodes

\section{Performance Analysis}

This section presents the simulation results to show the impact on energy consumption of different traffic applications like CBR (Constant bit rate), VBR (Variable bit rate) and FTP (File transfer protocol) in Zigbee home automation network having static IEEE 802.15.4 star topology.

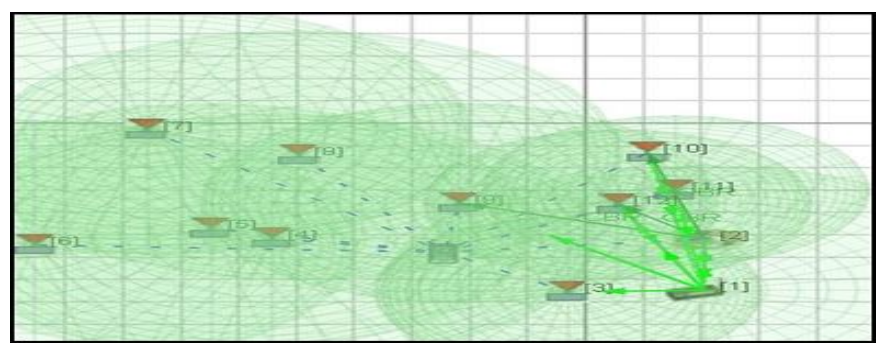

Figure 3. QualNet Animation during Simulation Execution

The following graphs depict comparison of different mobile device mode's (such as transmit, receive and idle mode) energy consumption with respect to different traffic types (such as CBR, VBR and FTP).

Figures 4 to 6 shows the value of energy consumed in transmit, receive and idle mode for different traffic types.

5.1. Effect of Different Traffic on Power Consumption in Transmit Mode: The energy consumed in transmit mode for FTP application is low with value 1.10 as compare to VBR and CBR with a value of 1.21. 


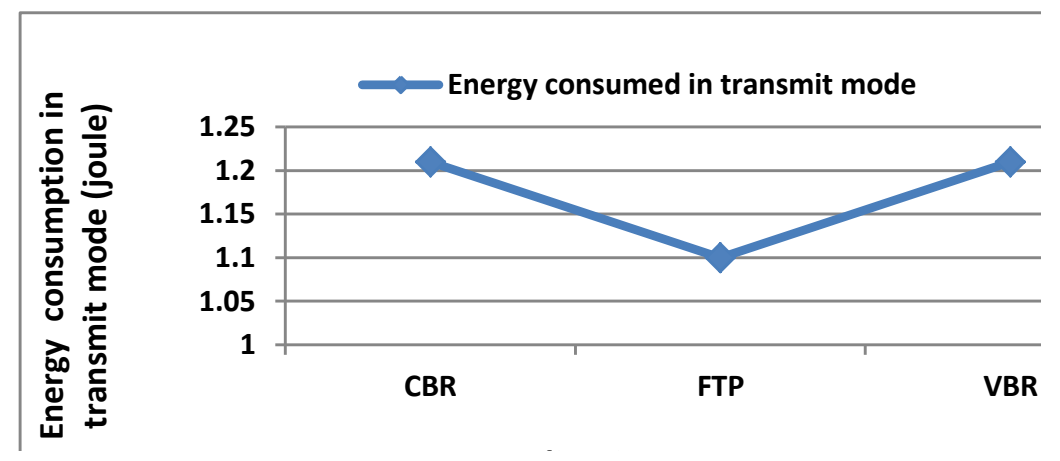

Figure 4. Energy Consumption in Transmit Mode v/s Different Traffic

5.2. Effect of Different Traffic on Power Consumption in Receive Mode: The energy consumed in receive mode for FTP application is also low with value 0.37 as compare to VBR and CBR with a value of 0.40 and 0.38 respectively.

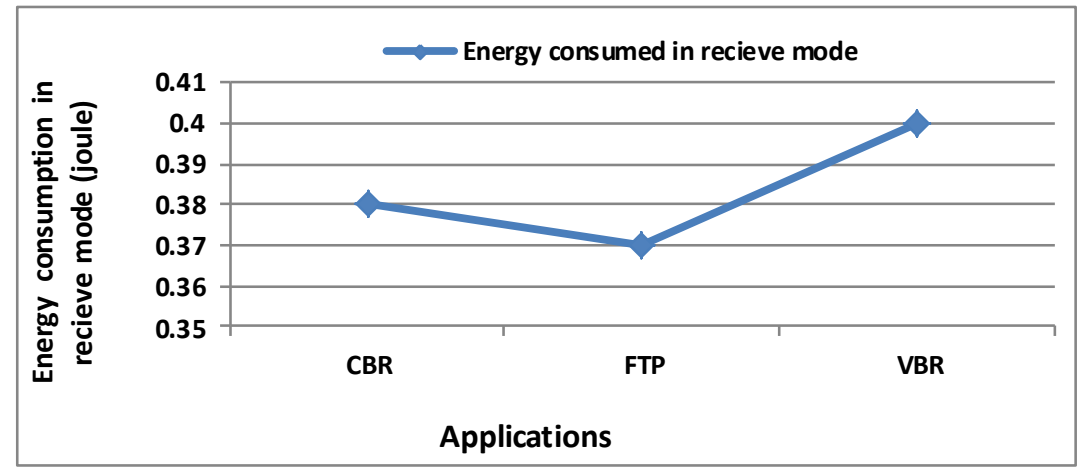

Figure 5. Energy Consumption in Receive Mode v/s Different Traffic

5.3. Effect of Different Traffic on Power Consumption in Idle Mode: The energy consumed in idle mode for FTP application is also low with value 3.78 as compare to VBR and CBR with a value of 3.81 and 3.80 respectively. So, FTP produced lowest value in transmit, receive and idle mode. In terms of energy consumption FTP overall performs better than other traffic application since low power consumption corresponds to high efficiency.

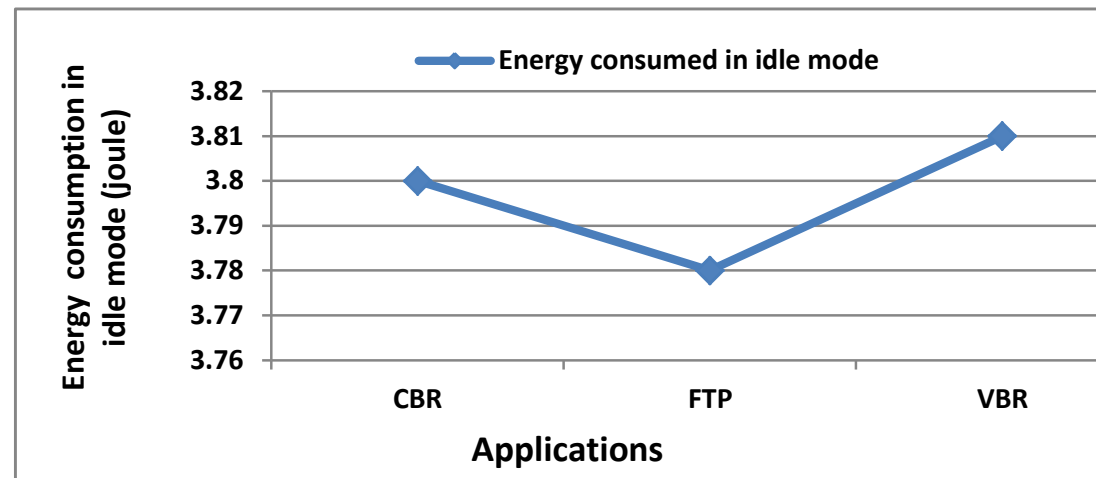

Figure 6. Effect of Different Traffic on Power Consumption in Idle Mode 
Table 1. Simulation Result of Effect of Different Traffic Types on Energy
Consumption

\begin{tabular}{|c|c|c|c|}
\hline Traffic Types & $\begin{array}{c}\text { Energy consumed in } \\
\text { transmit mode } \\
\text { ( joule) }\end{array}$ & $\begin{array}{c}\text { Energy consumed in } \\
\text { receive mode } \\
\text { ( joule) }\end{array}$ & $\begin{array}{c}\text { Energy } \\
\text { consumed in } \\
\text { idle mode } \\
\text { (joule) }\end{array}$ \\
\hline CBR & 1.21 & 0.38 & 3.80 \\
\hline FTP & 1.10 & 0.37 & 3.78 \\
\hline VBR & 1.21 & 0.40 & 3.81 \\
\hline
\end{tabular}

The Table 1 concludes, the effect on power consumption under different traffic applications in transmit, receive and idle mode of Zigbee network based home automation system.

\section{Conclusion}

This paper concludes the values of energy consumed in transmit, receive and idle mode for different traffic types is carried out. The results depicts that the energy consumed in transmit, receive and idle mode for FTP application is low with value 1.10, 0.37 and 3.78 as compare to VBR and CBR traffic. On other side, the VBR and FTP consumed energy with value of 1.21 at transmit mode, 0.40 and 0.38 at receive mode and 3.81 and 3.80 at idle mode respectively. So, FTP produced lowest value in transmit, receive and idle mode. In terms of energy consumption FTP overall performs better than other traffic application since low power consumption corresponds to high efficiency.

\section{References}

[1] IEEE 802.15.4, "Wireless Medium Access Control (MAC) and Physical Layer (PHY) Specifications for Low-Rate Wireless Personal Area Networks (LR-WPANs)", IEEE NY, (2003).

[2] Zigbee-Alliance: Zigbee specification, (2005).

[3] H. Seok Kim, J.-H. Song and S. Lee, "Energy-Efficient Traffic Scheduling in IEEE 802.15.4 for Home Automation Networks", IEEE Transactions on Consumer Electronics, vol. 53, no. 2, (2007), pp. 369-374.

[4] J. Zheng and M. J. Lee, "A comprehensive performance study of IEEE 802.15.4", Sensor Network Operations Book, IEEE Press, Wiley Interscience, Chapter 4, (2006), pp. 218-237.

[5] W. T. H. Woon and T. C. Wan, "Performance Evaluation of IEEE 802.15.4 Ad Hoc Wireless Sensor Networks: Simulation Approach”, IEEE International Conference on Systems, Man and Cybernetics, SMC '06, vol. 2, (2006), pp. 1443-1448.

[6] M. Kohvakka and M. Kuorilehto, "Performance Analysis of IEEE 802.15.4 and ZigBee for Large-Scale Wireless Sensor Network Applications”, PE- WASUN'06, Torremolinos, Malaga, Spain, (2006).

[7] J. Zheng and M. J. Lee, "A comprehensive performance study of IEEE 802.15.4", Sensor Network Operations Book, IEEE Press, Wiley Interscience, Chapter 4, pp. 218-237, (2006).

[8] B. Bougard, F. Catthoor, D. C. Daly, A. Chandrakasan and W. Dehaene, "Energy efficiency of IEEE 802.15.4 standard in dense wireless microsensor networks: modeling and improvement perspectives", Proceedings of Design, automation and test in Europe (DATE’05) (Munich, Germany, IEEE, vol. 1, (2005) March 7-11, pp. 196-201.

[9] G. Lu, B. Krishnamachari and C. S. Raghavendra, "Performance evaluation of the IEEE 802.15.4 MAC for low-rate low power wireless networks", Proceedings of the 23rd IEEE International Performance Computing and Communications Conference (IPCCC '04), Phoenix, Ariz, USA, (2004), pp. 701-706.

[10] N. F. Timmons and W. G. Scanlon, "Analysis of the performance of IEEE 802.15.4 for medical sensor body area networking", Proceedings of the 1st IEEE international conference, On Sensor and ad hoc communications and networks (SECON'04) (Santa Clara, CA, USA, IEEE, (2004), pp. 16-24. 
[11] J. Javier Garcia and T. Falck, "Quality of Service for IEEE 802.15.4 based Wireless Body Sensor Networks", 3rd International Conference on Pervasive Computing Technologies for Healthcare, (2009), pp. 1-6.

[12] G. Lu, B. Krishnamachari and C. S. Raghavendra, "Performance evaluation of the IEEE 802.15.4 MAC for low-rate low-power wireless networks", Proceedings of the 23rd IEEE international Performance computing and communications conference (IPCCC'04)", Phoenix, AZ, USA, IEEE, (2004) April 15-17, pp. 701-706.

[13] R. Veerendra1, "Performance Analysis of Low Rate and Low Power IEEE 802.15.4 Standard for Personal Wireless Area Networks", IJCSNS International Journal of Computer Science and Network Security, vol. 10, no. 11, (2010), pp. 233-238.

[14] R. Hoque Khan and F. Zepel, "RF-level Power Consumption Measurement (RF-PM) for Zigbee Wireless Sensor Network-Towards Cross Layer Optimization", IEEE, (2008), pp. 959-966.

[15] P. C. Ng, S. C. Liew, K. C. Sha and W. T. To, "Experimental Study of Hidden Node Problem in IEEE 802.11 Wireless Networks", Proc. IEEE SIGCOMM 2005, Philadelphia, PA, (2005).

[16] S. Mohanty and S. Kumar Patra, "Performance Analysis of Quality of Service Parameters for IEEE 802.15.4 Star Topology using MANET routing”, ICWET ‘10, Mumbai, Maharashtra, India, (2010).

[17] A. Ortega, "Variable Bit Rate Video Coding", university of southern California, Los Angeles, California, pp. $1-55$.

[18] O. Kamariotis, "Bridging the Gap between CBR and VBR for H264 Standard", World Academy of Science, Engineering and Technology, vol. 8, (2005), pp. 339-344.

[19] J. Postel, "File Transfer Protocol (FTP)" RFC: 959, (1985), pp. 1-37.

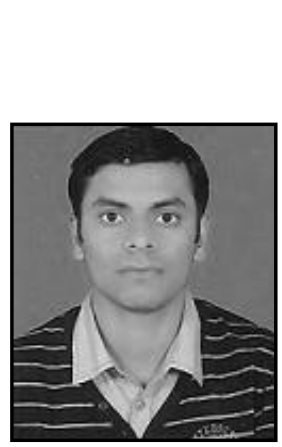

\section{Authors}

Kunal Malik received Bachelor of Technology degree in Electronics and Communication Engineering from Haryana College of technology \& management, Kaithal affiliated to Kurukshetra University Kurukshetra, India. The current focus of his research interests is on radio resource management, mobility management for next generation wireless networks.

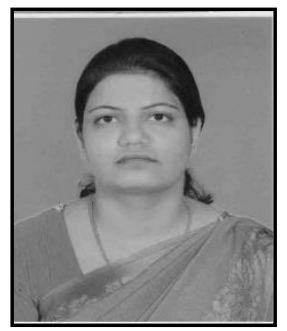

Kiran Ahuja has done outstanding research in the fields of optical and wireless communication. She received her B.Tech \& M.Tech degree in Electronics and Communication Engineering from Punjab Technical University, Jalandhar, India and pursuing $\mathrm{Ph}$. D in Electronics \& Communication Engineering in wireless communication and networks field. She worked as faculty at DAVIET, Jalandhar. 